EPJ Web of Conferences 60, 20036 (2013)

DOI: $10.1051 /$ epjconf/20136020036

(C) Owned by the authors, published by EDP Sciences, 2013

\title{
Search for new physics using events with two same-sign isolated leptons in the final state at CMS
}

\author{
S. Folgueras ${ }^{1}$ and M. Dünser ${ }^{2}$, on behalf of the CMS Collaboration \\ ${ }^{1}$ Universidad de Oviedo \\ ${ }^{2}$ ETH Zürich
}

\begin{abstract}
We present a search for new physics using events with two same-sign isolated leptons with the presence of $b$-jets in the final state, targetting SUSY scenarios by strong production of squarks and gluinos where the $3^{r d}$ generation squarks are lighter than other squarks. No excess above the standard model background is observed. The results are interpreted in various SUSY models, and we are able to exclude gluino (sbottom) masses up to 1000 (450) $\mathrm{GeV}$ at $95 \% \mathrm{CL}$.
\end{abstract}

\section{Introduction}

Events with same sign dilepton final states are very rare in the SM context, but they appear naturally in many different new physics scenarios such as SUSY where two same-sign dileptons can be produced in the decay chain of supersymetric particles.

For the sake of this analysis we will consider SUSY processes dominated by strong production of gluinos and squarks where $3^{\text {rd }}$ generation squarks are lighter than other squarks, resulting in an abundance of top and bottom quarks produced in the decay chain. We will assume that the SUSY decay chain ends with the LSP $\widetilde{\chi}_{1}^{0}$ ), that escapes undetected and therefore contribute strongly to the $E_{\mathrm{T}}^{\text {miss }}$ of the event.

In general, same-sign dileptons can be particularly sensitive to SUSY models with compressed spectra where the mass of the LSP is very close to the mass of the produced supersymetric particle, by requiring two same-sign leptons we can recover events that may skip other selections. We therefore search for SUSY using same sign dilepton events with hadronic activity and large $E_{\mathrm{T}}^{\text {miss }}$ and we interpret the results in the context of various SUSY models. What we present here is just a short summary of the analysis performed by CMS [1], more details can be found in the original publication [2].

\section{Event Selection}

We require two isolated same-sign leptons ( $e$ or $\mu$ ) with $p_{\mathrm{T}}>20 \mathrm{GeV}$, consistent with originating from the same vertex. Events are collected using dilepton triggers and an extra veto on the third lepton is applied to suppress Drell-Yan production. The isolation of the leptons is computed with particle-flow information, and an event-by-event correction is made to account for the effect of the multiple pp interaction in the same bunch crossing (pileup). This correction consists in substracting the estimated contribution from the pileup in the isolation cone. 
For the baseline selection we require the event to have at least two b-tagged jets (with $p_{\mathrm{T}}>40$ $\mathrm{GeV}$ ). The signal regions are defined by impossing tighter cuts on the number of (b) jets, scalar sum of the $p_{\mathrm{T}}$ of all identified jets $\left(H_{\mathrm{T}}\right)$ and $E_{\mathrm{T}}^{\text {miss }}$.

\section{Background estimation}

There are some sources of SM background to potential new physics signals: events with one or two fake leptons, opposite-sign events in which one of the electron charge has been badly measured and events with two same-sign prompt-leptons. A description of the relevance of these backgrounds and how they are estimated is presented in this section.

\subsection{Backgrounds with one or two fake leptons}

Backgrounds with one or two fake leptons, include processes such as semi-leptonic $\overline{\mathrm{t}}$ or $W+$ jets where one of the leptons comes from a heavy-flavor decay, misindentified hadrons, muons from lightmeson decay in flight, or electrons from unidentified photon conversions. We estimate this background starting from measuring the probability of a lepton being fake or prompt using a QCD or Z enriched sample respectively. We then apply those probabilities to events passing the full kinematic selection but in which one or two of the leptons fail the isolation requirements. About $40-50 \%$ of the total background is due to this processes and we assign a 50\% systematic uncertainty to account for the lack of estatistics in the control sample as well as the little knowledge we about about the control sample composition.

\subsection{Events with charge mis-identification}

These are events with opposite-sign isolated leptons where one of the leptons (typically an electron) and its charge is misreconstructed due to sever bremsstrahlung in the tracker materia (this effect is negligible for muons). We estimate this background by selecting opposite-sign $e e$ or $e \mu$ events passing the full kinematic selection, weighted by the probability of electron charge misassignment. This probability is measured in a $Z \rightarrow e e$ sample in data by simply calculating the ratio between samesign and opposite-sign events in such sample and it validated in $\mathrm{MC}$, this probability is of the order $0.02(0.2) \%$ for electrons in the barrel(endcap). This source of background only only accounts for the $5-10 \%$ of the total background. A $20 \%$ systematic uncertainty on this background is considered to account for the $p_{\mathrm{T}}$ dependence of the probability.

\subsection{Rare SM processes.}

These include SM processes that yield two same-sign prompt leptons, including $\bar{t} \bar{t} W, \bar{t} \bar{Z}, W^{ \pm} W^{ \pm}$ among others. These processes constitutes about 30-40\% of the total background.

All these background are obtained from Monte Carlo simualtions and we assign a 50\% systematic uncertainty to this background sources as we have very little knowledge on the cross-sections.

\section{Results}

The search is based on comparing observed and predicted yields in 8 signal regions with different requirements motivated by various possible new physics models. The definition of these search regions, as well as the observed and predicted yields are shown in Table 1. 95\% confidence level (CL) observed upper limit are also shown on this table. 

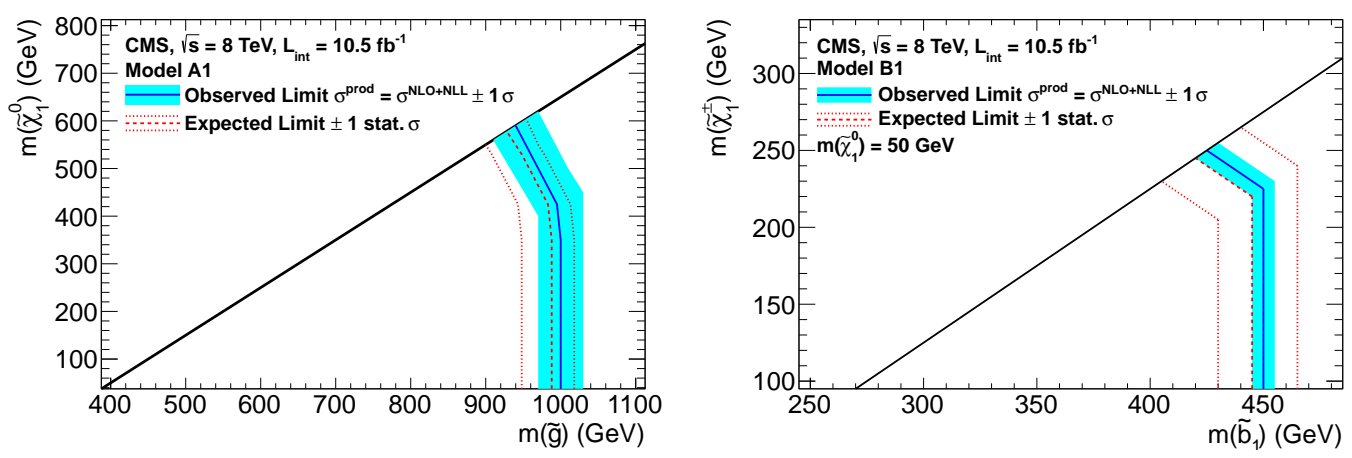

Figure 1. Exclusion (95\%C.L.) in the $m \widetilde{\chi}_{1}^{0}$ ) vs $m \widetilde{g}$ ) plane, where each of the gluinos decays $\widetilde{g} \rightarrow t \widetilde{\chi}_{1}^{0}$ with virtual stops (left). Exclusion (95\%C.L.) in the $m\left(\widetilde{\chi}_{1}^{ \pm}\right)$vs $m(\widetilde{b})$ plane, for a model with sbottom pair production. These models have more than two mass parameters, and cannot be fully represented in a two dimensional plot.

Table 1. A summary of the combination of results for this search. For each signal region (SR), we show its most distinguishing kinematic requirements, the prediction for the three background (BG) components as well as the total, and the observed number of events.

\begin{tabular}{|l|c|c|c|c|c|c|c|c|c|}
\hline & SR0 & SR1 & SR2 & SR3 & SR4 & SR5 & SR6 & SR7 & SR8 \\
\hline No. of jets & $\geq 2$ & $\geq 2$ & $\geq 2$ & $\geq 4$ & $\geq 4$ & $\geq 4$ & $\geq 4$ & $\geq 3$ & $\geq 4$ \\
No. of btags & $\geq 2$ & $\geq 2$ & $\geq 2$ & $\geq 2$ & $\geq 2$ & $\geq 2$ & $\geq 2$ & $\geq 3$ & $\geq 2$ \\
Lepton charges & $++/--$ & $++/--$ & ++ & $++/--$ & $++/--$ & $++/--$ & $++/--$ & $++/--$ & $++/--$ \\
$E_{\mathrm{T}}^{\text {miss }}$ & $>0 \mathrm{GeV}$ & $>30 \mathrm{GeV}$ & $>30 \mathrm{GeV}$ & $>120 \mathrm{GeV}$ & $>50 \mathrm{GeV}$ & $>50 \mathrm{GeV}$ & $>120 \mathrm{GeV}$ & $>50 \mathrm{GeV}$ & $>0 \mathrm{GeV}$ \\
$H_{\mathrm{T}}$ & $>80 \mathrm{GeV}$ & $>80 \mathrm{GeV}$ & $>80 \mathrm{GeV}$ & $>200 \mathrm{GeV}$ & $>200 \mathrm{GeV}$ & $>320 \mathrm{GeV}$ & $>320 \mathrm{GeV}$ & $>200 \mathrm{GeV}$ & $>320 \mathrm{GeV}$ \\
\hline Charge-misID BG & $3.35 \pm 0.67$ & $2.70 \pm 0.54$ & $1.35 \pm 0.27$ & $0.04 \pm 0.01$ & $0.21 \pm 0.05$ & $0.14 \pm 0.03$ & $0.04 \pm 0.01$ & $0.03 \pm 0.01$ & $0.21 \pm 0.05$ \\
Fake BG & $24.77 \pm 12.62$ & $19.18 \pm 9.83$ & $9.59 \pm 5.02$ & $0.99 \pm 0.69$ & $4.51 \pm 2.85$ & $2.88 \pm 1.69$ & $0.67 \pm 0.48$ & $0.71 \pm 0.47$ & $4.39 \pm 2.64$ \\
Rare SM BG & $11.75 \pm 5.89$ & $10.46 \pm 5.25$ & $6.73 \pm 3.39$ & $1.18 \pm 0.67$ & $3.35 \pm 1.84$ & $2.66 \pm 1.47$ & $1.02 \pm 0.60$ & $0.44 \pm 0.39$ & $3.50 \pm 1.92$ \\
\hline Total BG & $39.87 \pm 13.94$ & $32.34 \pm 11.16$ & $17.67 \pm 6.06$ & $2.22 \pm 0.96$ & $8.07 \pm 3.39$ & $5.67 \pm 2.24$ & $1.73 \pm 0.77$ & $1.18 \pm 0.61$ & $8.11 \pm 3.26$ \\
Event yield & 43 & 38 & 14 & 1 & 10 & 7 & 1 & 1 & 9 \\
\hline$N_{U L}(13 \%$ unc.) & 27.2 & 26.0 & 9.9 & 3.6 & 10.8 & 8.6 & 3.6 & 3.7 & 9.6 \\
$N_{U L}(20 \%$ unc.) & 28.2 & 27.2 & 10.2 & 3.6 & 11.2 & 8.9 & 3.7 & 3.8 & 9.9 \\
$N_{U L}(30 \%$ unc.) & 30.4 & 29.6 & 10.7 & 3.8 & 12.0 & 9.6 & 3.9 & 4.0 & 10.5 \\
\hline
\end{tabular}

None of the search regions shows any significant excess over the SM background predictions, therefore we set interpret the results in several physics models[2]. For example Figure 1 shows the exclusion regions for gluino-pair production decaying into on-shell stops as well as direct sbotton pair production. We are able to exclude gluino (sbottom) masses up to $1(0.45) \mathrm{TeV}$ with such models.

\section{Conclusions}

We have presented results of a search for new physics with events with same-sign dileptons using the CMS detector at the LHC. No significant deviations from the standard model expectations are observed. The results are used to set exclusion limits into several SUSY models. We are able to probe gluino (sbottom) masses up to $1(0.45) \mathrm{TeV}$.

\section{References}

[1] CMS Collaboration, JINST, JINST 3 S08004, (2008).

[2] CMS Collaboration, JHEP, JHEP03(2013)037, 1-30 (2013) 